\title{
The Tribe Sporoboleae (Gramineae) in Japan
}

By

\section{Jisaburo Ohwi.*}

Receivcd July 15, 1941.

Gen. 1. Garnotia Brongn. in Duperr. Voy. Bot. Coq. (1829) 132.

Sp. 1. Garnotia acutigluma (Steud.) OHwi, comb. nov.

Urachne acutigluma STEud. Synops. 1 (1854) 121; Miq. Prol. Fl. Japon. (1867) 164.

Garnota stricta (non Brongn.) OHwi in Act. Phytotax. et Geobot. 6 (1937) 149.

Nom. Jap. Ao-shiba.

Hab. Riukiu. Distrib. Java (?), New Guinea.

Gen. 2. Sporobolus R. Br. Prodr. (1812) 169.

1. Ammua $20 \mathrm{~cm}$ vel minus alta, folia radicalia inconspicua, foliorum laminae lanceolatae, margine cilis basi callosis longe ciliatae, 2-5 $\mathrm{cm}$ longae, antherae $1 / 3-1 / 2 \mathrm{~mm}$ longae.

.................... japonicus

1. Perennis, $15-80 \mathrm{~cm}$ alta, folia radicalia conspicua, laminae foliorum lineares, sine ciliis basi callosis.

2. Gluma $2^{\text {da }}$ quam lemma aequilonga, laminae foliorum $3-15 \mathrm{~cm}$ longae.

3. Estolonifera, culmus 1-3-nodis, foliorum laminae 5-10 em longae, spiculae paullo purpurascentes, $1.7-2 \mathrm{~mm}$ longae, antherae $1 / 2-2 / 3 \mathrm{~mm}$ longae. .....

.S. Hancei

3. Stolonifera, culmus 5-10-nodis, foliorum laminae invulutae, spiculae $2.5-3 \mathrm{~mm}$ longae, antherae $1-1.5 \mathrm{~mm}$ longae. ..................... virgicus

2. Gluma $2^{\mathrm{da}} 1 / 2-2 / 3$ lemmatis aequans, foliorum laminae $20-50 \mathrm{~cm}$ Iongae.

3. Ramuli pedicellique crassiusculi breves, rami dense spiculosi, $0.5-5 \mathrm{~cm}$ longi, spiculae $2-2.5 \mathrm{~mm}$ longae, antherae $1 / 2-2 / 3$ longae. ........... elongatus

3. Ramuli pedicellique tenues, rami subdense spiculosi $1-7 \mathrm{~cm}$ longi, spiculae $1.5-2 \mathrm{~mm}$ longae, antherae $1 / 2 \mathrm{~mm}$ longae. .................. diander

Sp. 2. Sporobolus japonicus (Steud.) Maxim. ex Rendle in Journ. Linn. Soc. 36 (1904) 388; NAKAI, Flor. Korean. 2 (1911) 355.

Agrostis japonicus Steud. Synops. 1 (1854) 171.

Sporobolus ciliatus (non PresL) Matsum. in Bot. Mag. Tokyo 11(1897) 444.

Sporobolus piliferus (non Kunth) Honda in Bot. Mag. Tokyo 49 (1926) 321 et Monogr. Poac. Japon. (1930) 201.

\footnotetext{
* Botanical Institute, Kyoto Imperial University, Kyoto.
} 
Sporobolus ciliatus var. japonicus (Steud.) Hack. in Bull. Herb. Boiss. 7 (1899) 648.

Nom. Jap. Hige-shiba.

Hab. Hondo, Shikoku, Kiushiu, Korea. Distr. China med.

Sp. 3. Sporobolus Hancei RendLe in Journ. Linn. Soc. 36 (1904) 387 ; OHwi in Act. Phytotax. et Geobot. 3 (1934) 81.

Nom. Jap. Hime-nezuminoo.

Hab. Formosa, Riukiu (ins. Okinawa). Distr. China austr.

Sp 4. Sporobolus virginicus (Linn.) Kunth, Rév. Gram. 1 (1829) 678 et Enum. Pl. 1 (1833) 210 ; Makino in Bot. Mag. Tokyo 10 (1896) 64; Hack. in Bull. Herb. Boiss. 7 (1899) 721 ; Rendee, l. c. (1904) 389 ; Hoxda, Monogr. (1930) 201; Hitchc. Man. Grass. U.S. (1935) 394 et 962 et Man. Grass. West Ind. (1936) 87.

Agrostis virginicus Linn. Sp. Pl. (1753) 63.

Nom. Jap. Sonare-shiba.

Hab. Formosa, Riukiu (ins. Miyako, Okinawa, Amami-oshima, Tokunoshima, Tanegashima), Bonins, praesertim in arenis littoris occurit. Distr. Pantrop.

Sp. 5. Sporobolus diander (Retz.) Beauv. Ess. Agrost. (1812) 26; Kunth, Enum. Pl. 1 (1833) 213; Hook. f. Fl. Brit. Ind. 7 (1897) 247; RENDLE in Journ. Linn. Soc. 36 (1904) 387; Honda, l. c. (1930) 200.

Agrostis diandra RETz. Obs. 5 (1789) 19.

Vilfa Retzii Steud. Synops. 1 (1854) 162.

Nom. Jap. Futashibe-nezuminoo.

Hab. Formosa, Riukiu (ins. Miyako, Okinawa, Tokunoshima) Distr. Malaya, India, China austr., Ins. maris pacific.

Sp. 6. Sporobolus elongatus R. BR. Prodr. (1810) 170; Kunth, Enum. Pl. 1 (1833) 212 et Suppl. (1835) 168; Miq. Prol. Fl. Japon. (1867) 166; Hack. in Bull. Herb. Boiss. 7 (1899) 648; Honda, Monogr. Poac. Japon. (1930) 200.

Cinna japonica Steud. Synops. 1 (1854) 182.

Agrostis fertilis STEud. l. c. 170.

Sporobolus indicus (vix R. BR.) Auct. I̊l. Jap.

Nom. Jap. Nezumino-o.

Hab. Hondo, Shikoku, Kiushiu, Riukiu, Formosa, Korea. Distr. Malaya, India, Australia.

var. purpureo-suff usus OHWI, var. nov.

Spica elongata, lemmate partim purpureo-suffuso differt.-Typus: Hondo: Mukoshima, urbe Kyoto (leg. K. Takeuchi, 1929). 
Nom. Jap. Murasaki-nezuminoo.

Hab. Crese. cum forma typica.

Gen. 3. Sphaerocaryum Nees ex Steud. Nom. Bot. ed. 2, 2 (1841) 620 ; Hook. f. Fl. Brit. Ind. 7 (1897) 246.

Steudelella Honda, Monogr. Poac. Japon. (1930) 258.

Sp. 7. Sphaerocaryum malaccense (Trin.) PILGer in Fedde Repert. 45 (1938) 2.

Panicum malaccense Trin. De Gram. Panic. (1826) 204.

Steudelella pulchella Honda, Monogr. (1930) 259, excl. syn.

Sphaerocaryum elegans Nees ex Steud. Nom. Bot. ed. 2, 2 (1841) 620 ; Hook. f. Fl. Brit. Ind. 7 (1897) 246: ReNdLE in Journ. Linn. Soc. 36 (1904) 387.

Panicum barbivaginale Hayata, Mater. Fl. Formos. (1911) 400.

Nom. Jap. O-ushikusa.

Hab. Formosa. Distr. India, Malaya, China austr., Philipp.

Gen. 4. Aristida Linn. Sp. Pl. (1753) 82 et Gen. ed. 5 (1754) 35.

1. Arista crassa patens contorta, basi in columnam tortam connata, gluma $\mathbf{1}^{\mathrm{ma}}$ trinervis 6-10 mm longa, gluma $2^{\mathrm{da}} 1$-nervis $10-13 \mathrm{~mm}$ longa, plantae perennes.

2. Foliorum laminae plicatae, $0.3-0.6 \mathrm{~mm}$ diam, fere teretes, antherae $1 \mathrm{~mm}$ longae.

2. Foliorum laminae plicatae, explic. $1.5 \mathrm{~mm}$ latae, striatae. ........... boninensis

1. Arista tenuis erecta vel media tantum patens, non contorta, basi non connata, gluma $1^{\text {ma }}$ 1-nervis.

2. Perennis, panicula effusa laxa, rami elongati patentes, spiculae ascendentes vel adpressae pallidae, gluma $1^{\mathrm{ma}} 10-11 \mathrm{~mm}$ longa, gluma $2^{\text {da }} 6-8 \mathrm{~mm}$ longa, antherae

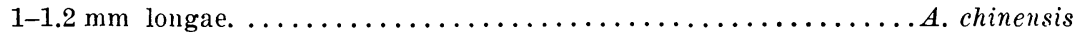

2. Annua, panicula contracta angusta, rami appressi breves, spiculae saepe purpurascentes, gluma $1^{\mathrm{ma}} 4-6 \mathrm{~mm}$ longa, $2^{\mathrm{da}}$ paullo longior, arista media basi abrupte geniculata, patens, lateralis brevis erecta. ........... longcspica

Sp. 8. Aristida Takeoi Oнwi in Bot. Mag. Tokyo 45 (1931) 182; Henrard, Rev. Aristid. suppl. (1933) 741.

Nom. Jap. O-matsubashiba.

Hab. Riukiu (Ins. Okinawa, et ? Amami-oshima).

Sp. 9. Aristida boninensis Orw et Tuyama in Bot. Mag. Tokyo 51 (1937) 126.

Aristida Cumingiana (non Trin. et Rupr.) NakaI in Bot. Mag. Tokyo 25 (1911) 223.

Nom. Jap. Matsuba-shiba.

Hab. Bonins.

Sp. 10. Aristida chinensis Munro in Proc. Amer. Acad. 4 (1860) 
363 ; Benth. Fl. Hongk. (1861) 427 ; RendLe in Journ. Linn. Soc. 36 (1904) 381 ; Henrard, 1. c.

Aristida formosana Honda in Bot. Mag. Tokyo 41 (1927) 379 et Monogr. Poac. Japan. (1930) 211.

Nom. Jap. Taiwan-matsubashiba.

Hab. Formosa (Shinchiku, leg. Y. ShImada!). Distr. China austr.

Sp. 11. Aristida longespica PoIr. in Lam. Encycl. suppl. 1 (1810) 452 ; Hiтchc. Man. Grass. U.S. (1935) 446 et 799.

Nom. Jap. Hime-matsubashiba.

Hab. Hondo, introducta in urbe Morioka. Distr. Amer. bor.

Gen. 5. Muhlenbergia Schreb. Gen. Pl. (1789) 44, sine nom. specif. et in Gmel. Syst. Nat. 2 (1791) 87 et 171.

1. Culmi basi decumbentes ramosi estoloniferi, glumae $1 / 3-2 / 3$ lemmatis aequilongae, antherae $0.5-1 \mathrm{~mm}$ longae.

2. Glumae late ovatae obtusulae ca. $1 / 3$ lemmatis aequantes, arista longa, antherae $0.5-0.7 \mathrm{~mm}$ longae, eulmi basi serius ramosi. ............... arisanensis

2. Glumae anguste ovatae acutae $1 / 2-2 / 3$ lemmatis aequilongae, antherae $0.7-1 \mathrm{~mm}$ longae, arista longiuscula, culmi basi pauci-ramosi. .............. japonica

1. Culmi basi erecti, stolones longos squamis aphyllis dense tectos emittentes, glumae $1 / 4-4 / 5$ lemmatis aequantes.

2. Glumae ovatae obtusae vel subotusae, saepe margine superne denticulatae, $1 / 4-1 / 3$ lemmatis aequantes, culmi elati, panicula saepe effusa, arista elongata, antherae $0.7-0.9 \mathrm{~mm}$ longae. ........................................

2. Glumae lanceolatae acuminatae vel acutissimae, integrae, $3 / 5-4 / 5-$ lemmatis aequantes, culmi $40-120 \mathrm{~cm}$ alti, panicula saepe angusta.

3. Spiculae $2.5-3 \mathrm{~mm}$ longae, glumae $3 / 5-2 / 3$ lemmatis aequantes, culmi superne erecti vel nutantes, serius ramosi, basi stolones longos squamis molliuseulis adpressis dorso vix inflatis obtectos emittentes, antherae $0.5-0.7 \mathrm{~mm}$ longae.

4. Culmi etiam superne erecti, sursum serius ramosi. .........M. ramosa

4. Culmi superne nutantes vel incumbentes, ad nodos ramosi...M. incumbens

3. Spiculae $3-4 \mathrm{~mm}$ longae, glumae $2 / 3-4 / 5$ lemmatis aequilongae, culmi erecti vix superne ramosi, basi stolones longos squamis coriaceis dorso valde inflatis convexis obtectos emittente, antherae $0.8-2 \mathrm{~mm}$ longae.

4. Spiculae $3-3.5 \mathrm{~mm}$ longae, paniculae rami ascendentes, antherae 0.8-1.2 $\mathrm{mm}$ longae, culmi subtenues, foliorum laminae $3-7 \mathrm{~mm}$ latac, stolonibus

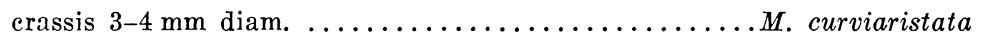

4. Spiculae 4-4.5 mm longae, paniculae rami adpressi, antherae $1.5-2 \mathrm{~mm}$ longae (rarissime $1.3 \mathrm{~mm}$ ), culmi tenues, foliorum laminae 2-4 $\mathrm{mm}$ latae, stolonibus tenuibus ca. $2 \mathrm{~mm}$ diam. ................. hakonensis

Sp. 12. Muhlenbergia arisanensis Hayata, Icon. Pl. Formos. 7 (1918) 87.

Muhlenbergia Hügelii (non Trin.) Honda, Monogr. (1930) 215, partim.

Nom. Jap. Arisan-nezumino-o.

Hab. Formosa, in montibus. Distr. Philipp., Nova Guinea. 
Sp. 13. Muhlenbergia japonica Steun. Synops. 1 (1854) 422, errore japanica; Rendde in Journ. Linn. Soc. 36 (1904) 385; Honda, Monogr. Poac. Japon. (1930) 217; Miyabe et Kudo, Fl. Hokk. a. Saghal. 2 (1931) 129; Roshev. in Komar. Fl. URSS. 2 (1934) 121; Kitag. Lineam. Fl. Manshuriae (1939) 84.

Nom. Jap. Nezumi-gaya.

Hab. Yezo austr.-occid., Hondo, Shikoku, Kiushiu, Korea. Distr. Reg. temp. Asiae orient.

Sp. 14. Muhlenbergia Huegelii Trin. in Mém. Acad. St. Pétersb. 6:6:2 (1841) 47 et 293; Steud. Synops. 1 (1854) 178; Rendle in Journ. Linn. Soc. 36 (1904) 385; Honda, Monogr. Poac. Japon. (1930) 215, excl. pl. e Formos.; Miyabe et Kudo, Fl. Hokk. a. Saghal. 2 (1931) 129 ; Rosirev. in Komar. Fl. URSS. 2 (1934) 121.

Muhlenbergia viridissima NeEs ex STEud. Synops. 1 (1854) 178; Kourar. Fl. Manshur. 1 (1901) 269.

Nom. Jap. O-nezumigaya.

Hab. Yezo, Hondo, Shikoku, Kilishiu, Korea. Distr. India, China, Manchuria, Amur.

Sp. 15. Muhlenbergia ramosa ( $\mathrm{H}_{\mathrm{ACK}}$.) MaKino in Jouri. Jap. Bot. 1 (1917) 13; Honda, Monogr. Poac. Japon. (1930) 217.

Muhlenbergia japonica var. ramosa HAck. in Bull. Herb. Boiss. 7 (1899) 647.

Nom. Jap. Kidachino-nezumigayá.

Hab. Hondo media et oceid.

Sp. 16. Muhlenbergia incumbens Honda in Bot. Mag. Tok! 40 (1926) 320 et Monogr. Poac. Japon. (1930) 216.

Nom. Jap. Yabu-nezumigaya.

Hab. Kiushiu, ?Hondo(m. Ogasayama in Totomi, leg. D. Sumbzu !?).

Sp. 17. Muhlenbergia curviaristata (OHwi) OHwi, comb. nov.

Muhlenbergia ramosa var. curviaristata OHwi in Act. Phytotax. et Geobot. 6 (1937) 292.

A $M$. ramosa differt, culmo simplici vel vix ramoso, spiculis majoribus 3-3.5 mm longis, glumis sterilibus $1 / 4^{-1 / 3}$ brevioribus, squamis stolonum valde inflato-convexis rigidulis, antheris ca. $1 \mathrm{~mm}$ longis.

Nom. Jap. Koshino-nezumigaya.

Hab. Hondo media.

var. nipponica OHwi, var. nov.

Muhlenbergia hatonensis (vix Makino) Honda, Monogr. Poac. Japon. (1930) 214, partim. 
Differt a typo, arista recta quam spicula 2-3-plo longiore.--Typus: Hondo: Sobudake in Tajima (Y. Araki n. 1094).

Nom. Jap. Miyama-nezumigaya.

Hab. Yezo, Kuriles austr., Hondo.

Sp. 18. Muhlenbergia hakonensis (Hack.) Makivo in Journ. Jap. Bot. 1 (1917) 13; Honda, l. c. (1930) 214, ex pte.

Muhlenbergia japonica var. hakonensis HAck. in Bull. Herb. Boiss. 7 (1899) 647.

Nom. Jap. Tachi-nezumigaya.

Hab. Hondo, Shikoku, Kiushiu, Korea (Ins. Quelpaert).

\section{本邦産ねづみのを族 (和文摘要)}

$$
\text { 大 井次 三 郎 }
$$

StAPF † C. E. HubBard 八此族トシテハ Sporobolus (及ビ米國產) Blepharoneuron）ダケヨ認メテ居ル。篗者八 Roshevitz 二從ツテ Sphaerocaryum, Huhlenbergia ヨ含メ夕外, 更二後者二似夕 Aristidla 及ビ多少離レテハ居ルガ Garnotia 7 モ本族二加へタガ, Brachyelytrum 八ムシロBromus 二近イト考へルノデ除外シタ。 熱帶及南溫帶 鄉土トシ, Eragrosteae 二相當スル一小花ノ族デ本邦二八五岿十八種 ガ認メラレル。 